\title{
Biological therapy of inflammatory bowel disease
}

\author{
Danuta Owczarek, Dorota Cibor, Małgorzata Szczepanek, Tomasz Mach \\ Department of Gastroenterology and Hepatology, Jagiellonian University, Medical College, Kraków, Poland
}

\section{KEY WORDS}

biological therapy, Crohn's disease, ulcerative colitis
Correspondence to:

Danuta Owczarek, MD, PhD, Klinika Gastroenterologii i Hepatologii, Uniwersytet Jagielloński, Collegium Medicum,

ul. Śniadeckich 5, 31-531 Kraków, Poland, phone: + 48-12-424-73-40, fax: + 48-12-424-73-80 e-mail: owczarek@su.krakow.pl Received: August 18, 2008

Revision accepted: October 10, 2008

Conflict of Interest: none declared.

Pol Arch Med Wewn. 2009; 119 (1-2): 84-89

Translated by Iwona Rywczak, $\mathrm{MD}, \mathrm{PhD}$

Copyright by Medycyna Praktyczna, Kraków 2009

\section{ABSTRACT}

Ulcerative colitis (UC), Crohn's disease (CD) and indeterminate colitis are defined as inflammatory bowel diseases (IBD). Those diseases involve disorders of numerous immunological mechanisms associated with cellular and humoral immune response. In CD cellular response is considered to be of crucial importance, and dominant cytokines include: tumor necrosis factor a (TNF-a), interferon $\gamma$ (INF- $\gamma$ ) and interleukins $1 \beta$ (IL-1 $\beta$ ), IL-2, IL-6, IL-8, IL-12. In UC, increased expression of Th2 (responsible for humoral response) is observed. It is connected with increased production of interleukins: 4 (IL-4), IL-5, IL-6, IL-10 and TNF-a. Lack of balance between pro-inflammatory and anti-inflammatory cytokines is of vital importance in pathogenesis of IBD. Conventional therapy of CD and UC quite commonly fails to bring satisfactory results, therefore an interest in new therapeutic options, that is, biological therapy, gene therapy, hematopoietic stem cell transplantation, and leucapheresis, has aroused recently. Biological therapy is focused on different stages of the inflammatory process. The fundamentals of biological strategy involve neutralization of pro-inflammatory cytokines, use of anti-inflammatory cytokines and inhibition of neutrophil adhesion. Biological therapy is a promising option because it enables to withdraw corticosteroids and immunosuppressive agents or to reduce their dose. Moreover, it shortens the hospital stay, allows to avoid surgical procedures, extends the remission period and improves patients' quality of life. Currently, 2 agents, infliximab and adalimumab, are registered for the biological therapy of $C D$ in Poland.

INTRODUCTION Ulcerative colitis (UC), Crohn's disease (CD) and indeterminate colitis are defined by a common term of inflammatory bowel disease (IBD). These diseases have common complex and multifactorial pathogenesis. It is currently accepted that genetic, environmental and immunological factors contribute to development of IBD. ${ }^{1,2}$ IBD involves disorders of numerous immunological mechanisms associated with cellular and humoral immune response. It is believed that in $C D$, the cellular response, with increased activation of CD $4+\mathrm{T}$ helper (Th) cells mostly differentiated into Th1 subpopulation, is of great significance. This lymphocyte population produces increased amounts of cytokines, which have a significant impact on the further course of the immune response. Predominant cytokines in CD are tumor necrosis factor- $\alpha$ (TNF- $\alpha$ ), interferon- $\gamma$ (INF- $\gamma$ ) and interleukins $1 \beta$ (IL- $1 \beta)$, IL-2, IL-6, IL-8 and IL-12. UC involves increased expression of Th2 cells engaged in the humoral response, what is associated with production of interleukin 4 (IL-4), IL-5, IL-6, IL-10 and TNF $\alpha .^{3-5}$ In IBD pathogenesis, the imbalance between pro-inflammatory cytokines (IL-1, IL-1 $\beta$, IL-2, IL-6, IL-8, IL-12, IL-17, IL-23, TNF- $\alpha$, INF- $\gamma$ ) and anti-inflammatory cytokines (IL-4, IL-10, IL-11, IL-13) is also important. In recent studies performed in the murine model using monoclonal antibodies, attention was also paid to IL-17 and IL-23. It leads to the conclusion that these cytokines have a relevant function in the pathogenesis of $\mathrm{UC}$ and $\mathrm{CD} .^{6}$

An important phenomenon in the inflammatory process of IBD is an increased expression of endothelial leukocyte adhesion molecule 1 (ELAM-1) and intercellular adhesion molecule 1 (ICAM-1) on the surface of endothelial cell membranes. The increased expression of those adhesion molecules on capillary endothelium results 
in a higher number of plasmocytes, lymphocytes, macrophages and neutrophils within the inflamed tissue.

Biological therapy is a type of treatment aimed at various stages of the inflammatory process. Basic directions of biological therapy involve neutralization of pro-inflammatory cytokines, use of anti-inflammatory cytokines and inhibition of neutrophil adhesion. ${ }^{7,8}$ Currently, infliximab and adalimumab are 2 biological drugs licensed for $C D$ therapy in Poland. Other preparations are currently tested in clinical trials and are not routinely used in IBD therapy; however, they include preparations licensed for therapy of other disorders, for example rheumatoid arthritis or multiple sclerosis.

Indications for biological therapy in inflammatory bowel disease Indications for biological therapy in $\mathrm{CD}$ are as follows:

1 induction therapy in patients with moderate or severe disease activity who do not respond to conventional treatment with 5-ASA preparations, glucocorticosteroids and/or immunosuppressive agents

2 induction therapy in patients with fistulas, present despite appropriate standard therapy (antibiotic therapy, immunosuppression, surgical drainage)

3 maintenance treatment in patients who responded to the induction therapy.

In UC, biological therapy is recommended as induction therapy in patients with active UC who did not respond to standard treatment (5-ASA preparations, glucocorticosteroids, immunosuppressive agents), and as maintenance treatment in patients who responded to induction therapy.

The former IBD treatment algorithm, used for several years, is based on a "step-up" strategy that involves a gradual enhancement of the therapy by adding glucocorticosteroids to 5-ASA preparations and, with no improvement, an immunosuppressive agent, and only ultimately a biological drug. ${ }^{9}$ Currently, a "top-down" strategy, in which IBD treatment is initiated with a biological drug, is recommended still more commonly. ${ }^{10}$

Clinical trials aiming at assessment of efficacy and safety of biological therapy of duration of several years and also the "top-down" method in IBD are still being used. Doubts about the duration of biological therapy with the "top-down" strategy are associated with increased risk of adverse events. ${ }^{11,12}$

Despite numerous trials focusing on biological therapy, duration of maintenance therapy has not been determined to date, but, as the trial analysis shows, it may last up to one year. ${ }^{13}$

Contraindications for biological therapy in inflammatory bowel disease Contraindications for biological therapy are hypersensitivity to the active or any ancillary agent, active tuberculosis or other severe infections, like sepsis and opportunistic infections, a history of malignant disease or active cancer, significant immunization observed prior to the disease onset, demyelinating diseases, and moderate to severe cardiac insufficiency (New York Heart Association [NYHA] class III/IV).

Biological therapy could not be initiated in patients with active (including chronic or local) infection, until the infection has been successfully treated. Before the initiation of the therapy, all patients should be examined in order to exclude both active and inactive (latent) infections. If latent tuberculosis is diagnosed before the initiation of biological therapy, appropriate prophylaxis should be implemented. In such cases, possible benefits of biological therapy should be carefully reassessed. Diagnostic evaluation of hepatitis should also be made before treatment initiation. Clinical trials using TNF- $\alpha$ antagonists demonstrated hepatitis reactivation in patients with chronic hepatitis B virus (HBV) infection. HBV carriers, who require biological therapy, should be carefully monitored throughout the whole period of therapy and several months afterwards. If $\mathrm{HBV}$ infection reactivates, biological therapy should be discontinued and antiviral therapy, e.g. with lamivudin or entacavir, should be introduced. Currently, there is no sufficient data concerning treatment of HBV carriers with antiviral agents combined with TNF- $\alpha$ antagonists, to prevent $\mathrm{HBV}$ reactivation. Likewise, there is no evidence regarding secondary transmission of infection with live vaccines in patients receiving biological agents; therefore, this group of patients may simultaneously receive vaccines, excluding live vaccines.

Biological therapy should be discontinued in patients with heart failure NYHA class I/II who demonstrated new symptoms or whose cardiac insufficiency symptoms worsened. Particular precautions should also be taken in biological therapy in patients with chronic obstructive pulmonary disease and with demyelinating disorders of the central nervous system.

Controlled clinical trials on TNF- $\alpha$ blockers showed higher prevalence of lymphomas in patients receiving anti-TNF- $\alpha$ antibodies as compared to the control group. However, the prevalence was low and the follow-up period for patients receiving placebo was shorter than for patients undergoing biological therapy. According to the current opinions, a risk of lymphoma or other type of cancer cannot be excluded in patients receiving anti-TNF- $\alpha$ antibodies. Based on currently available evidence, it is not yet clear whether TNF- $\alpha$ blockers influence the risk of mucous membrane dysplasia and colorectal cancer. Therefore, in patients with newly diagnosed dysplasia of colon mucosa undergoing biological therapy, risks and benefits for each patient should be thoroughly assessed and therapy cessation should be considered.

Biological therapy is not recommend ed in pregnant women. Clinical experience is too limited to exclude the risk of an adverse 
effect of anti-TNF- $\alpha$ antibodies on an appropriate immune response in newborns. Patients in child-bearing age undergoing biological therapy are recommended to take contraceptives throughout the therapy period and up to 6 months afterwards. Biological therapy is also not recommended during breastfeeding.

Adverse effects in biological therapy The most common adverse effects during biological therapy are as follows:

1 acute and delayed transfusion reactions

2 delayed reactions, like serum disease

3 lupus-like syndrome

4 reactivation tuberculosis

5 bacterial and fungal infections (aspergillosis, histoplasmosis, cryptococcosis, candidosis, listeriosis, pneumocystosis)

6 increased activity of liver enzymes

7 cardiovascular insufficiency

8 development of cancer - lymphomas (rare)

9 mortality similar to untreated patients.

Prevalence and types of adverse events are associated with development of antibodies against TNF- $\alpha$ blockers. For infliximab, this phenomenon is observed in $6-13 \%$ of patients and for adalimumab only in $2.6 \%$. It has been found that simultaneous use of immunosuppressive agents (azathioprine, 6-mercaptopurine) or methotrexate blocks the synthesis of anti-infliximab and anti-adalimumab antibodies.

Role of tumor necrosis factor- $a$ in the inflammatory process TNF- $\alpha$ is produced mainly by monocytes and macrophages, and to a lesser extent by neutrophils, keratinocytes, fibroblasts and mastocytes. Factors stimulating TNF- $\alpha$ secretion include endotoxins, antigens and osmotic stress. Production and release of TNF- $\alpha$ are stimulated, among others, by INF- $\gamma$ and IL-1. Physiologically, TNF- $\alpha$ occurs in 2 forms: as a precursor, $26 \mathrm{kDa}$ transmembrane protein and the soluble (free) form of molecular mass of $17 \mathrm{kDa}$. The soluble form is responsible for most of biological functions of TNF- $\alpha$, while the transmembrane form is active in processes, like apoptosis, cell proliferation, activation of B lymphocytes, or inflammation.

There are 2 types of receptors for TNF- $\alpha$ : type 1 (p55 protein) and type 2 ( $\mathrm{p} 75$ protein) of molecular mass $55 \mathrm{kDa}$ and $75 \mathrm{kDa}$, respectively. They differ in the glycosylation degree and affinity to TNF- $\alpha$, therefore, they may transmit different signals to the cell.

TNF- $\alpha$ triggers a cascade of pro-inflammatory reactions stimulating production of numerous cytokines and has significance both in induction of the inflammatory process and its sustaining. It is a main cytokine involved in the pathomechanism of autoimmune diseases, transplant rejection and septic shock. At the molecular level, it activates a nuclear factor responsible for control of transcription of pro-inflammatory cytokine genes. At the tissue level, TNF- $\alpha$ stimulates production of cell adhesion molecules (e.g. ICAM, ELAM, integrins) by endothelial cells, leading to increased penetration of lymphocytes, macrophages and neutrophils from the circulation into inflamed tissues, angiogenesis, fibroblast proliferation, development of granulomas and in creased prothrombic activity. TNF- $\alpha$ stimulates production of platelet activating factor (PAF) and IL- 8 by endothelial cells and induces local production of leukotriene $\mathrm{B}_{4} \cdot{ }^{14,15}$ Stimulation of the production and secretion of IL- 1 and IL- 6 influences the development of acute phase reaction with release of C-reactive protein (CRP) and with such symptoms as fever, anemia, thrombocytopenia, leukocytosis, weight loss. TNF- $\alpha$ is also responsible for evoking pain by increasing sensitivity of nociceptors to prostaglandins.

Biological agents licensed for inflammatory bowel diseases treatment Infliximab Is a chimeric human/murine monoclonal IgG1 antibody, binding with high affinity both to the soluble and the transmembrane form of human TNF- $\alpha$, however does not neutralize the lymphotoxin form (TNF- $\beta$ ). Infliximab quickly forms stable complexes with human TNF- $\alpha$. This is tantamount to loss of biological activity by TNF- $\alpha$.

The drug is administered in $5 \mathrm{mg} / \mathrm{kg}$ doses by 2 -hour intravenous infusion. Induction therapy in 3 doses is recommended, following the algorithm of week 0, 2 and 6, and for sustaining the remission in a dose repeated every 8 weeks. Available evidence does not justify further infliximab therapy in patients who have failed to respond to induction therapy. ${ }^{16,17}$

Adalimumab is a recombinant human monoclonal antibody obtained by expression in Chinese hamster ovary cells. Adalimumab is a fully human immunoglobulin G1. It specifically binds to TNF- $\alpha$ and neutralizes its biological function, blocking TNF- $\alpha$ interaction with p55 and p75 receptors on the cell surface. It also modulates the biological response, induced and regulated by TNF- $\alpha$, that involves among others in altered levels of intercellular adhesion molecules responsible for leukocyte migration (ELAM-1, ICAM-1, vascular cell adhesion molecule-1 [VCAM-1]).

The drug is administered in induction therapy in a dose of $80 \mathrm{mg}$ by subcutaneous injection, and then $40 \mathrm{mg}$ in week 2 . If a quick response to treatment is required, higher doses could be administered, i.e. $160 \mathrm{mg}$ a week (a dose could be administered as 4 injections during $24 \mathrm{~h}$ or 2 injections daily during 2 subsequent days), and then $80 \mathrm{mg}$ in week 2. In order to sustain the remission, $40 \mathrm{mg}$ is administered every second week. Clinical trials showed that in patients who did not respond to treatment within 4 weeks, continuation of maintenance treatment up to week 12 inclusive may be beneficial. In patients who do not respond to treatment within that time, continuation of such treatment should be reconsidered. ${ }^{18,19}$ 
Biological agents licensed for other disorders or undergoing clinical trials Etanercept Is a genetically modified human protein made by combining 2 ligands of human $\mathrm{p} 75 \mathrm{TNF}-\alpha$ receptor with the Fc region of human immunoglobulin G1. Etanercept binds 2 TNF- $\alpha$ molecules, acting as a "false" receptor, and, in contrast with infliximab, it also neutralizes the TNF lymphotoxin form TNF- $\beta$.

Clinical trials involved $25 \mathrm{mg}$ doses subcutaneously, twice a week, in patients with active $C D$ for 12 weeks. Clinical and endoscopic improvement was achieved in about $50 \%$ of the patients, but it has lasted on average for approximately 3 weeks since the drug was withdrawn. It may result both from short half-life of the medication $(68 \pm 12 \mathrm{~h})$, and from the fact that etanercept blocks only circulating forms of TNF- $\alpha .^{20,21}$ Etanercept is licensed for treatment of active rheumatoid arthritis, psoriatic arthritis, juvenile idiopathic arthritis and ankylosing spondylitis.

Onercept Is a recombined form of human soluble p55 TNF receptor. A study by Rutgeerts et al. involved 207 patients with active CD who were receiving onercept subcutaneously in several different doses or placebo 3 times a week for 8 weeks. After 8 weeks, remission was achieved in $23.5 \%$ of the patients in the placebo group and in $34.8 \%, 20.0 \%, 26.1 \%$, and $28.6 \%$ of the patients in the groups receiving onercept (10 mg, $25 \mathrm{mg}, 35 \mathrm{mg}$ and $50 \mathrm{mg}$ doses, respectively). ${ }^{22}$ Other completed randomized placebo-controlled trials demonstrated good tolerance of onercept, but its lower effectiveness in patients with active $\mathrm{CD}$ in comparison with infliximab, adalimumab or certolizumab pegol. ${ }^{23,24}$

CDP 571 (Humicade) is a humanized, monoclonal anti-TNF- $\alpha$ IgG4 antibody, made in 95\% of human immunoglobulin and in $5 \%$ of murine protein. CDP 571 neutralizes both the soluble and membrane forms of human TNF- $\alpha$. Clinical trials using CDP 571 in CD were initiated simultaneously with the trials with infliximab were published in 1997 . The trials showed effectiveness of CDP 571 in induction of the disease remission and its good tolerance. ${ }^{25}$ In 2004, results of the trial performed in the Mayo Clinic were published. This trial involved CDP 571 in $10 \mathrm{mg} / \mathrm{kg}$ doses infused intravenously, each 8th week during 24 weeks in active CD patients. Clinical response was assessed in weeks 2 and 28. In week 2, clinical response was achieved in $49.5 \%$ (the group receiving CDP 571), and in the placebo group in $15.5 \%$ of the patients, whereas in week 28 , in $28.7 \%$ and $12.1 \%$ of the patients, respectively. It was found that CPD571 was effective in inducing only a short remission so it could not be used in a long-term therapy. ${ }^{26}$ In clinical trials on steroid-dependent $C D$ patients, with Crohn's disease activity index $\leq 150$, CDP517 was used in $10 \mathrm{mg} / \mathrm{kg}$ intravenous doses, once every 8 weeks during 36 weeks. Glycocorticosteroid dose reduction was possible in $29.3 \%$ of the patients treated with CDP517 and in $36.7 \%$ in the placebo group. The drug was found well tolerated but ineffective in glucocorticosteroid dose reduction in steroid-dependent $C D$ patients. ${ }^{27}$

Comparison of the trials using CDP 571 and infliximab found that CDP-571 was a safe medication, but not as effective in $C D$ as infliximab. Therefore, further clinical trials on CDP 571 in this disease were discontinued.

Certolizumab pegol (CDP 870) Is a monoclonal antibody, combining the Fab fragment of human anti-TNF- $\alpha$ antibody with polyethylene glycol. The combination with polyethylene glycol resulted in prolonged serum half-life to approximately 2 weeks, which permits less frequent dosage.

In the completed Phase II clinical trials, CDP 870 was used in doses of 100, 200, $400 \mathrm{mg}$ administered subcutaneously each 4 weeks. Af ter 12 weeks clinical improvement was observed in $29.7 \%, 30.6 \%$ and $33.3 \%$ of the patients, respectively, and in $15.1 \%$ of the patients in the placebo group. ${ }^{28}$ It was also shown that clinical response was more favorable in patients receiving certulizumab in doses of $400 \mathrm{mg}$, in whom CRP levels were above $10 \mathrm{mg} / 1 .{ }^{29,30}$ More favorable clinical response in induction of the remission was observed in the trials in which doses of $400 \mathrm{mg}$ CDP870 were additionally administered in week 2. ${ }^{31}$ It was also demonstrated that Certolizumab may be effective in treatment of active $C D$ patients who were refractory to infliximab therapy. ${ }^{32}$

In Poland and other countries, certolizumab has not been licensed yet. Phase III of PRECISE trials is still under way.

Golimumab (CNTO 148) Is a human monoclonal anti-TNF- $\alpha$ antibody. In the published phase III clinical trials, CNTO 148 was administered subcutaneously in doses of $50 \mathrm{mg}$ or $100 \mathrm{mg}$ every 2 or 4 weeks in patients with rheumatoid arthritis. Golimumab was found well tolerated and effective in patients who suboptimaly responded to methotrexate monotherapy. ${ }^{33}$ Centocor phase III clinical trials using golimumab every 4 weeks in subcutaneous doses of $50 \mathrm{mg}, 100 \mathrm{mg}$ and $200 \mathrm{mg}$ in patients with moderate and severe exacerbation of UC are currently underway.

Biological therapy also involves pro-inflammatory interleukin antibodies, like daclizumab, basiliximab (anti-IL-2 antibodies), atlizumab, tocilizumab (anti-IL-6 receptor antibodies), or antibodies against IL-12, IL-17 and IL-23. Attempts are also made to administer anti-inflammatory interleukins, including recombinant IL-10 and IL-11. Clinical trials are also conducted using antibodies against INF- $\gamma$ (fontolizumab), $\alpha_{4} \beta_{7}$-integrin (MLN-02), $\alpha_{4}$-integrin (natalizumab), CD3 lymphocytes (visilizumab), and alicaforsen (ISIS-2302 - antisense oligonucleotide against ICAM-1 that inhibits migration of monocytes and granulocytes to the inflammatory site).

Another direction of biological therapy in clinical trials is focused on the use of growth factors. 
Namely, treatment of UC patients involved epidermal growth factor in the form of rectal infusions, while treatment of $C D$ patients involved granulocyte colony stimulating factor and granulocyte-macrophage colony stimulating factor.

It should also be emphasized that IBD treatment involves also other therapeutic options such as gene therapy, hematopoietic stem cell transplantation or leukapheresis.

SUMIMARY Conventional CD and UC therapy quite commonly does not bring satisfactory results; therefore, interest in new treatment methods has been growing recently. Biological therapy is a highly promising prospect, since it enables to discontinue the use of glucocorticosteroids and immunosuppressives or their dose reduction, shortens the hospitalization period, allows to avoid surgical treatment, extends the remission period and improves the patient's quality of life.

A multidirectional character of biological therapy requires further clinical trials to demonstrate which treatment method is most beneficial in long-term follow-up. Currently, using anti-TNF- $\alpha$ monoclonal antibodies seems most promising.

\section{REFERENCES}

1. Abreu MT, Sparrow MP. Translational research in inflammatory bowe disease. Mt Sinai J Med. 2006; 78: 1067-1072.

2 Hendrickson BA, Gokhale R, Cho JH. Clinical aspects and pathophysiology of inflammatory bowel disease. Clin Microbiol Rev. 2002; 15: 79-94.

3 Podolsky DK. Inflammatory bowel disease. N Engl J Med. 2002; 347: 417-429.

4 Fiocchi C. Inflammatory bowel disease: etiology and pathogenesis. Gastroenterology. 1998; 115: 182-205.

5 Shanahan F. Inflammatory bowel disease; immunodiagnostics, immunotherapeutic and ecotherapeutics. Gastroenterology. 2001; 120: 622-636.

6 Yen D, Cheung J, Scheerens $H$, et al. Interleukin-23 is essential for $T$ cell-mediated colitis and promotes inflammation via interleukin-17 and interleukin 6. J Clin Invest. 2006; 116: 1310-1316.

7 Kurtovic J, Segal I. Recent advances in biological therapy for inflammatory bowel disease. Trop Gastroenterol. 2004; 25: 9-14.

8 Sandbory WJ, Targan SR. Biologic therapy in inflammatory bowel disease. Gastroenterology. 2002; 122: 1592-1608.

9 Desilva S, Kaplan G, Panaccione R. Sequential therapies for Crohn's disease: optimizing conventional and biologic strategies. Rev Gastroenterol Disord. 2008; 8: 109-116.

10 Armuzzi A, De Pascalis B, Fedeli P, et al. Infliximab in Crohn's disease: early and long-term treatment. Dig Liver Dis. 2008; 40: 271-279.

11 Shergill AK, Terdiman JP. Controversies in the treatment of Crohn's disease: the case for an accelerated step-up treatment approach. World $J$ Gastroenterol. 2008; 14: 2670-2677.

12 Caviglia R, Boskoski I, Cicala M. Long-term treatment with infliximab in inflammatory bowel disease: safety and tolerability issues. Expert Opin Drug Saf. 2008; 7: 617-632.

13 Bartnik W. [Guidelines for inflammatory bowel disease management]. Gastroenterol Pol. 2007; 14: 3-13. Polish.

14 Van Deventer SJH. Tumor necrosis factor and Crohn's disease. Gut. 1997; 40: 443-448.

15 Armstrond AM, Gardiner KR, Kirk SJ, et al. Tumor necrosis factor and inflammatory bowel disease. Br J Surg. 1997; 84: 1051-1058.

16 Present $\mathrm{DH}$, Rutgeerts $\mathrm{P}$, Targan $\mathrm{S}$, et al. Infliximab for the treatment of fistulas in patients with Crohn's disease. N Engl J Med. 1999; 340 1398-1405.

17 Hanauer SB, Feagan BG, Lichtenstein GR, et al. Maintenance infliximab for Crohn's disease: the ACCENT I randomized trial. Lancet. 2002 359: 1541-1549.

18 Hanauer SB, Sandborm WJ, Rutgeerts $P$, et al. Human anti-tumor necrosis factor monoclonal antibody (adalimumab) in Crohn's disease: the CLASSIC-1 trial. Gastroenterology. 2006; 130: 323-333.
19 Colombel JF, Sandborm WJ, Rutgeerts P, et al. Adalimumab for maintenance of clinical response and remission in patients with Crohn's disease: the CHARM trial. Gastroenterology. 2007; 132: 52-65

20 D'Haens GD, Swijsen C, Norman M, et al. Etanercept in the treatment of active refractory Crohn's disease: Asingle center pilot trial. Am J Gastroenterol. 2001; 96: 2564-2568.

21 Sandborn WJ, Hanauer SB, Katz S, et al. Etanercept for active Crohn's disease: a randomized, double-blind, placebo-controlled trial. Gastroenterology. 2001; 121: 1088-1094.

22 Rutgeerts P, Sandborn WJ, Fedorak RN, et al. Onercept for moderate - to severe Crohn's disease: a randomized, double-blind, placebo-controlled trial. Clin Gastroenterol Hepatol. 2006; 4: 888-893.

23 Rutgeerts P, Lemmens L, Van Assche G, et al. Treatment of active Crohn's disease with onercept (recombinant human soluble p55 tumor necrosis factor receptor): results of a randomized, open-label, pilot study. Aliment Pharmacol Ther. 2003; 17: 185-192.

24 Osterman MT, Lichtenstein GR. Current and future anti-TNF therapy for inflammatory bowel disease. Curr Treat Options Gastroenterol. 2007; 10: 195-207.

25 Stak WA, Mann SD, Roy AJ, et al. Randomised controlled trial of CDP571 antibody to tumor necrosis factor-alpha in Crohn's disease. Lancet. 1997; 349: 521-524.

26 Sandborn WJ, Feagan BG, Radford-Smith G, et al. CDP571, a humanised monoclonal antibody to tumour necrosis factor alpha, for mod erate to severe Crohn's disease: a randomised, double blind, placebo controlled trial. Gut. 2004; 53: 1485-1493.

27 Feagan BG, Sandborn WJ, Lichtenstein G, et al. CDP571, a humanized monoclonal antibody to tumour necrosis factor-alpha, for steroid-dependent Crohn's disease: a randomized, double-blind, placebo-controlled trial. Aliment Pharmacol Ther. 2006; 1: 617-628.

28 Schreiber S, Rutgeerts P, Fedorak R, et al. A randomized, placebo controlled trial of certolizumab pegol (CDP870) for treatment of Crohn's dis ease. Gastroenterology. 2005; 129: 807-814.

29 Rutgeerts P, Schreiber S, Feagan B, et al. Certolizumab pegol, a monthly subcutaneously administered Fc-free anti-TNF alpha, improves health-related quality of life in patients with moderate to severe Crohn's disease. Int J Colorectal Dis. 2008; 23: 289-296.

30 Schreiber S, Khaliq-Kareemi M, Lawrance IC, et al. Maintenance therapy with certolizumab pegol for Crohn's disease. N Engl J Med. 2007; 19: 296-298

31 Blick SK, Curran MP. Certolizumab pegol: in Crohn's disease. Bio Drugs. 2007; 21: 195-201.

32 Abreu MT. Crohn's disease in patients who fail infliximab therapy: what does the future hold? Rev Gastroenterol Disord. 2007; 7: 20-26.

33 Kay J, Matteson EL, Dasgupta B, et al. Golimumab in patients with active rheumatoid arthritis despite treatment with methotrexate: a random ized, double-blind, placebo-controlled, dose-ranging study. Arthritis Rheum. 2008: 58: 964-975 